\title{
Combination of Digital Image Processing and Statistical Data Segmentation to Enhance SPR and SPRi Sensor-responses
}

\section{Gleice M. de Aguiar}

Universidade Federal Rural do Semi-Arido

Leandro C. de Souza

Universidade Federal da Paraiba

Daniel F. L. de Souza

Universidade Federal da Paraiba

Leiva Casemiro Oliveira ( $\nabla$ leiva.casemiro@ufersa.edu.br)

Universidade Federal Rural do Semi-Arido https://orcid.org/0000-0001-9147-4604

\section{Research Article}

Keywords: Thin-film devices, Optical transducers, surface plasmon resonance spectroscopy, bootstrap, SPRi

Posted Date: November 10th, 2021

DOI: https://doi.org/10.21203/rs.3.rs-1001535/v1

License: (c) (i) This work is licensed under a Creative Commons Attribution 4.0 International License. Read Full License

Version of Record: A version of this preprint was published at Plasmonics on January 26th, 2022. See the published version at https://doi.org/10.1007/s11468-022-01604-z. 


\title{
Combination of digital image processing and statistical data segmentation to enhance SPR and SPRi sensor- responses
}

\author{
Gleice M. de Aguiar ${ }^{1}$, Leandro C. de Souza², Daniel F. L. de Souza ${ }^{3}$, and Leiva C. \\ Oliveira $^{1}$
}

Gleice M. de Aguiar and Leiva C. Oliveira

Department of Computer Science,

Universidade Federal Rural do Semi-Árido (UFERSA)

59625-900, Mossoró-RN, Brasil

Tel.: +55-83-999425759

E-mail: leiva.casemiro@ufersa.edu.br

Corresponding author

Leandro C. de Souza

Centers of Informatics

Universidade Federal da Paraíba (UFPB)

João Pessoa-PB, Brasil

Daniel F.L. de Souza

Centers of Informatics

Universidade Federal da Paraíba (UFPB)

Rio Tinto-PB, Brasil

\begin{abstract}
The work reports the combination of basics digital image processing (DIP) techniques and statistical segmentation strategy (SDS) to improve surface plasmon resonance curve (SPRC) and SPR imaging (SPRi) sensors' performance. The SPR image is used for sensing and monitoring biological events in the so-called SPR imaging process. In the traditional SPR process based on the attenuated total reflection (ATR) method, the image is used to create the SPR curve, and the curve features tracking is employed on sensing applications. The SPR curve features are enhanced after the pixels of the SPR image have been processed with lowcomplexity filters in the spatial domain (brightness, contrast, threshold, and morphological). The bootstrap was used as a statistical processing approach, selecting lines and columns from the image that was less affected by imperfections and noises in the image detector, and consequently reducing the SPR sensor instrumentation disturbances. Experimental tests with reversible binding water-mixture were performed, and both image and statistical processing were reported. The combination of DIP and SDS approaches improves the extraction of the curve features, increasing the performance in terms of resonance position sensitivity until $81 \%$.
\end{abstract}

Keywords - Thin-film devices; Optical transducers; surface plasmon resonance spectroscopy; bootstrap; SPRi. 


\section{INTRODUCTION}

Surface Plasmons Resonance (SPR) is a promising physical phenomenon for the development of instruments and tools to be applied for sensing biological events. Extremely sensitive to refractive index variations of the substances under analysis [1,2], the SPR sensors monitor the resonance position through the minimum reflectivity value obtained when illuminating a multilayer structure. Typically, the attenuated total reflection (ATR) configuration is employed [1,2], with a multilayer composed of a thin metal slab between two dielectric mediums, one being an optically transmissive material and the other being an analyzed substance [3].

A 1D or 2D image detector captures the reflected light after going through the multilayer. The generated image is used directly for sensing in the so-called SPR imaging process (SPRi) [4,5] or is graphically constructed previously before sensing, forming the SPR curve (SPRc). In both cases, the quality of the measurement is related to the image quality provided by the SPR sensor. As an example, 2D image samples with and without SPR excitation are present in Fig. 1. In the resonance condition, a low energy region is noticed and indicates the resonance position according to the substance refractive index. The SPRc and SPRi sensors track this position/intensity for sensing proceedings.
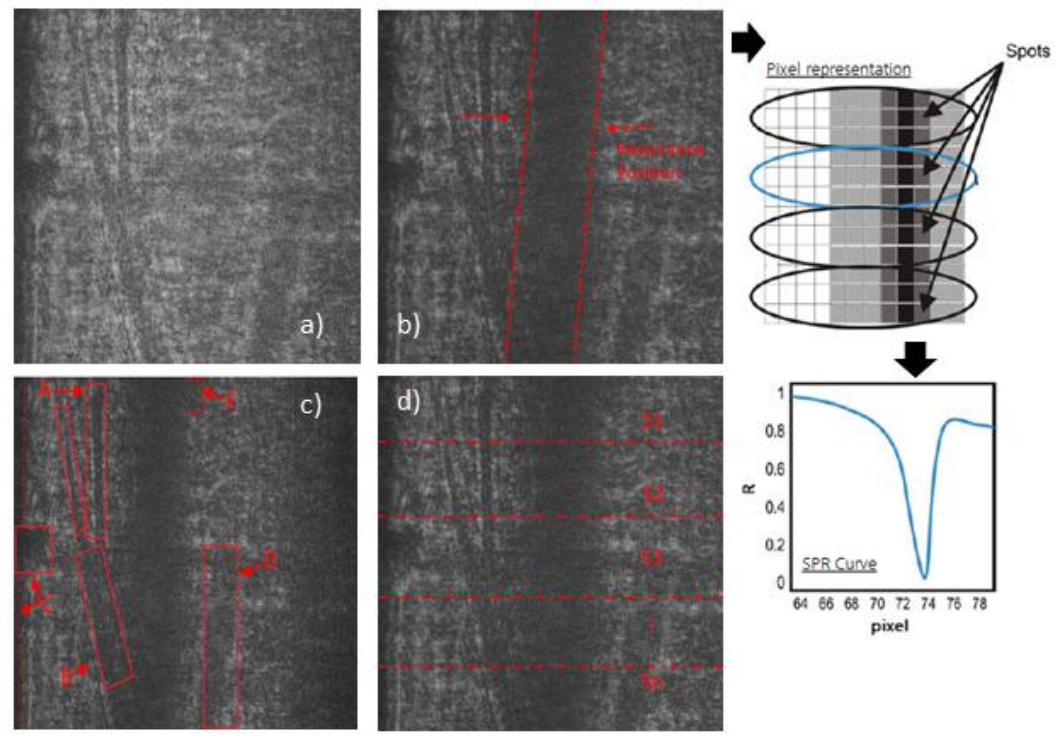

Figure 1 - Image a) without and b) with surface plasmon excitation and resonance position indication. Details for the image Pixel representation (SPRi) and the corresponding SPR curve. c) Examples of degradations/imperfections in SPR image. d) Logical spots regions obtained with the image segmentation.

The imperfections and noise of the experimental set-up of the SPR sensor are present in the generated image. As indicated in Fig. 1c, scratches (A), stains \& shading (B), mechanical misalignment (C), false minimum/resonance (D) and non-uniform response (E) deform the SPR image/SPR curve, causing detection of the resonance position difficult. In addition, the arising noises during the SPR sensor usage, related to the processes of transformation, generation, conversion, amplification, and digitization of incident light are also expressed in the SPR image. Brightness, contrast, and sharpness issues are commonly noticed in SPR images.

Algorithms to improve SPRc data extraction [6], curve characteristics enhancement [7], and for better selection of the region-of-interest (ROI) in SPRi $[8,9]$ are early reports. Attempts to model and remove image sensor noise [10], with machine learning approach [11], or based on transformation domain operation in SPR images for intensity processing [12] and noise removal [13] can be found elsewhere. Transformation domain operation, with non-linear filter as notch, fan-sector, and specific point filters [14] can be also applied for SPR image enhancement. In addition, the subdivision/segmentation of the SPR image into multiple spots can mitigate the non-uniformity of the image detector since the imperfections are spatially distributed in the 
detector area. Improvements in the signal-to-noise ratio [7] and in the dynamic response of the SPR sensor [15] can be obtained. As illustrated in Fig. 1d, the image can be segmented into several spot-regions ( $\mathrm{Si}$ ) to obtain the best $\mathrm{SPR}$ curve/SPRi ROI. In general, this is a process performed manually $[3,15]$ or through complex methods $[9,16]$ that require elaborate calibration and parameter acquisition procedures for the segmentation model.

Here we introduced a spatial domain digital image processing (DIP) combined with statistical data segmentation (SDS) approach for enhancing both SPRc and SPRi. Traditional enhanced, smooth and morphological operators are applied in the pixel matrix of the image. Next, the bootstrap method [17] is applied in the processed image to resample the pixel distribution of the SPRi/SPRc ROI. The approach is demonstrated with reversible binding solution at angular interrogation mode SPR operation. The new sensor performance indicated $81 \%$ improvement in the resonance angle $\left(\theta_{R}\right)$ shift measurement.

\section{EXPERIMENTAL REPORT}

The SPR sensor based on the well-know PPBIO prism [18,19] was used to obtain the SPR images. The experimental setup is shown in Fig. 2. The PPBIO has a trapezoidal structure, in which the light beams enter and exit parallel to the prism, avoiding rotational parts. The metallic layer is deposited directly on the prism, avoiding gel/oil for coupling. The PPBIO is capable of operating in the spectral interrogation (WIM) and angular (AIM) modes [19]. In AIM mode the PPBIO provides an angular range of $62^{\circ}$ to $73^{\circ}$, ideal for measuring aqueous substances. The sensor has a cylindrical lens LJ190L1 (for focusing) and a plano-convex spherical lens LA1951 (for collimating the light beams); the 670+/-10nm laser diode L670P005 and the two-dimensional CMOS camera DCC1645C with $1024 \mathrm{X} 1280$ pixels resolution, all commercialized by Thorlabs. The Acquisition, Control, Processing and Display (UACPD) unit embedded the combined DIP and SDS proposed approach.

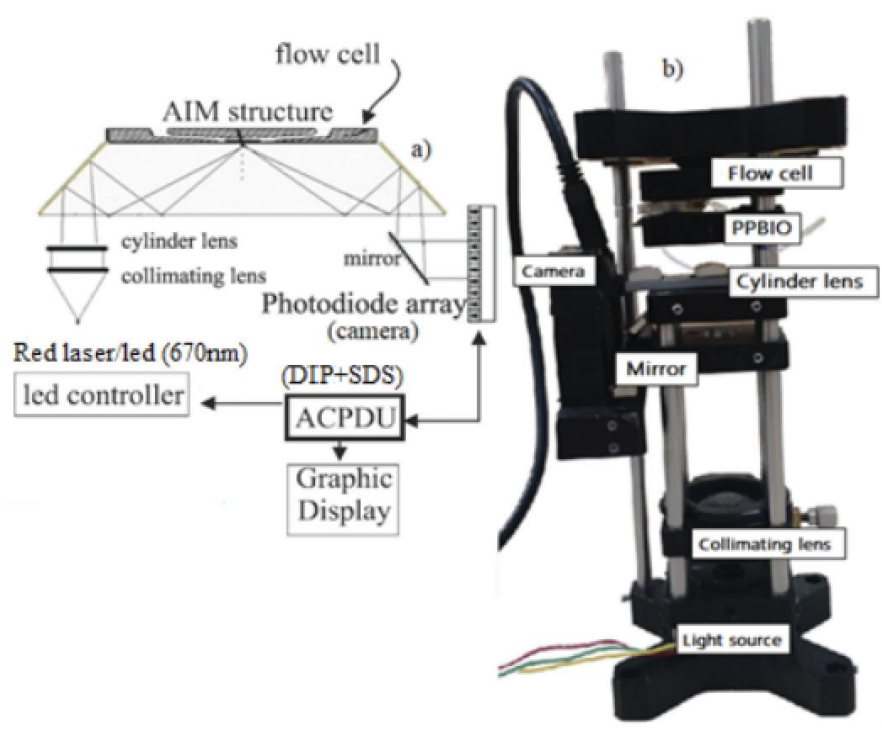

Figure 2 - a) Schematic of the SPR sensor based on PPBIO for the AIM mode and with a 2D CMOS camera. b) Photographic of the experimental setup. All components indicated in the figure.

The experiments have carried out with BK7 glass PPBIO, covered with a thin 50nm gold layer using the sputtering DC Cryofox Explorer 600LT from Polyteknik. The substances deionized and degassed water $\left(\mathrm{H}_{2} \mathrm{O}\right)$ with electrical resistance of $18.7 \mathrm{M} \Omega / \mathrm{cm}$ obtained with the Milipore Direct Q3 system, and solutions of PBS (Phosphate Buffered Saline) and Hypochlorite 
(Hypo.) were used. The substances have the following refractive index (RIU): $\mathrm{H}_{2} \mathrm{O}=1.33001$, $\mathrm{PBS}=1.33151$ and Hypo $=1.34501$.

The SPR images for each substance are shown in Fig. 3. Color or gray scale images can be used without compromising sensor performance. Substance with a higher refractive index shifts the resonance position to the left so that the minimum intensity of reflected light is located at the initial pixels. The pixel-angle relation [3] used for sensor calibration is $\theta(p)=-0.007 p+$ 72.765 , where $\mathrm{p}$ is the pixel position.

\section{SPATIAL DOMAIN PROCESSING OF SPR IMAGE}

The raw SPR images were processed with methods for adjusting brightness \& contrast, segmentation by a threshold, and noise/imperfections smooth with erosion and dilation morphology methods. References in the literature for the mathematical formulations of these techniques and simple implementation algorithms can be found elsewhere [14]. Fig. 4 presents the DIP application on the images of each substance and their respective SPR curves. Visual inspection, SPR curve depth, and minimum position were evaluated. Corrections differences in intensity level (brightness) and between the upper and lower levels (contrast) are necessary for compatibility between light source power (mA), camera integration time (ms) and ambient light (nW or MW). In general, SPR images have low average intensity (dark images) due to low power applied in order to avoid noise and fluctuations in the laser/led output [20]. Thus, the brightness and contrast operations aim to increase the intensity of the image's pixels.
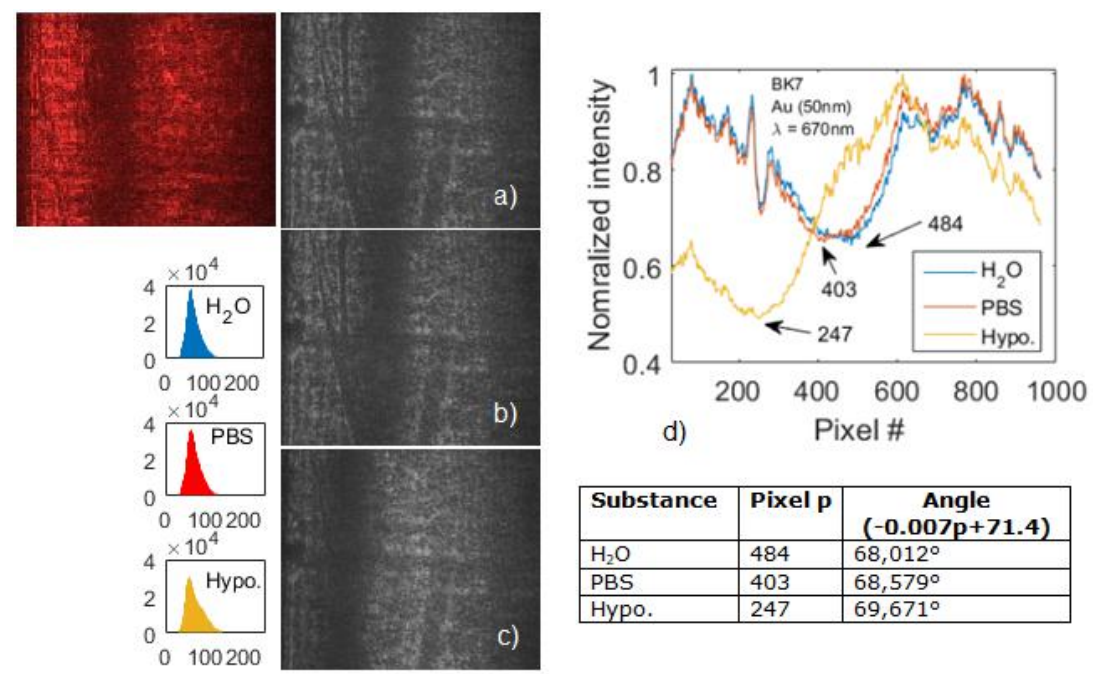

\begin{tabular}{|l|l|l|}
\hline Substance & Pixel p & $\begin{array}{c}\text { Angle } \\
(-\mathbf{0 . 0 0 7 p + 7 1 . 4 )}\end{array}$ \\
\hline $\mathrm{H}_{2} \mathrm{O}$ & 484 & $68,012^{\circ}$ \\
\hline PBS & 403 & $68,579^{\circ}$ \\
\hline Hypo. & 247 & $69,671^{\circ}$ \\
\hline
\end{tabular}

Figure 3 - SPR images for each substances. Color or gray scale images can be used. Histograms detailed of each grays scale images. a) $\mathrm{H}_{2} \mathrm{O}$ (color and gray scale), b) PBS and c) Hypo SPR images (SPRi). d) Respective SPR curves (SPRc) and the pixel-angle relation.

Segmentation threshold was applied above to the pixel average intensity, in opposite processing to brightness \& contrast. Despite visually enhancing the resonance region and the depth of the SPR curve did not change. Furthermore, morphological processing was evaluated. Erosion reduces or sharpens objects in an image, whereby details smaller than the structuring element are filtered out (removed) from the image. Thus pixels-area shrink in size, and holes within those areas (resonance position) become larger. Unlike erosion, dilation enlarges or thickens objects in an image. With a 10x10 pixel square structuring element, a $38 \%$ depth increase approximately was obtained with the erosion method, maintaining the characteristics of width, asymmetry, and slope of the curve [3]. 

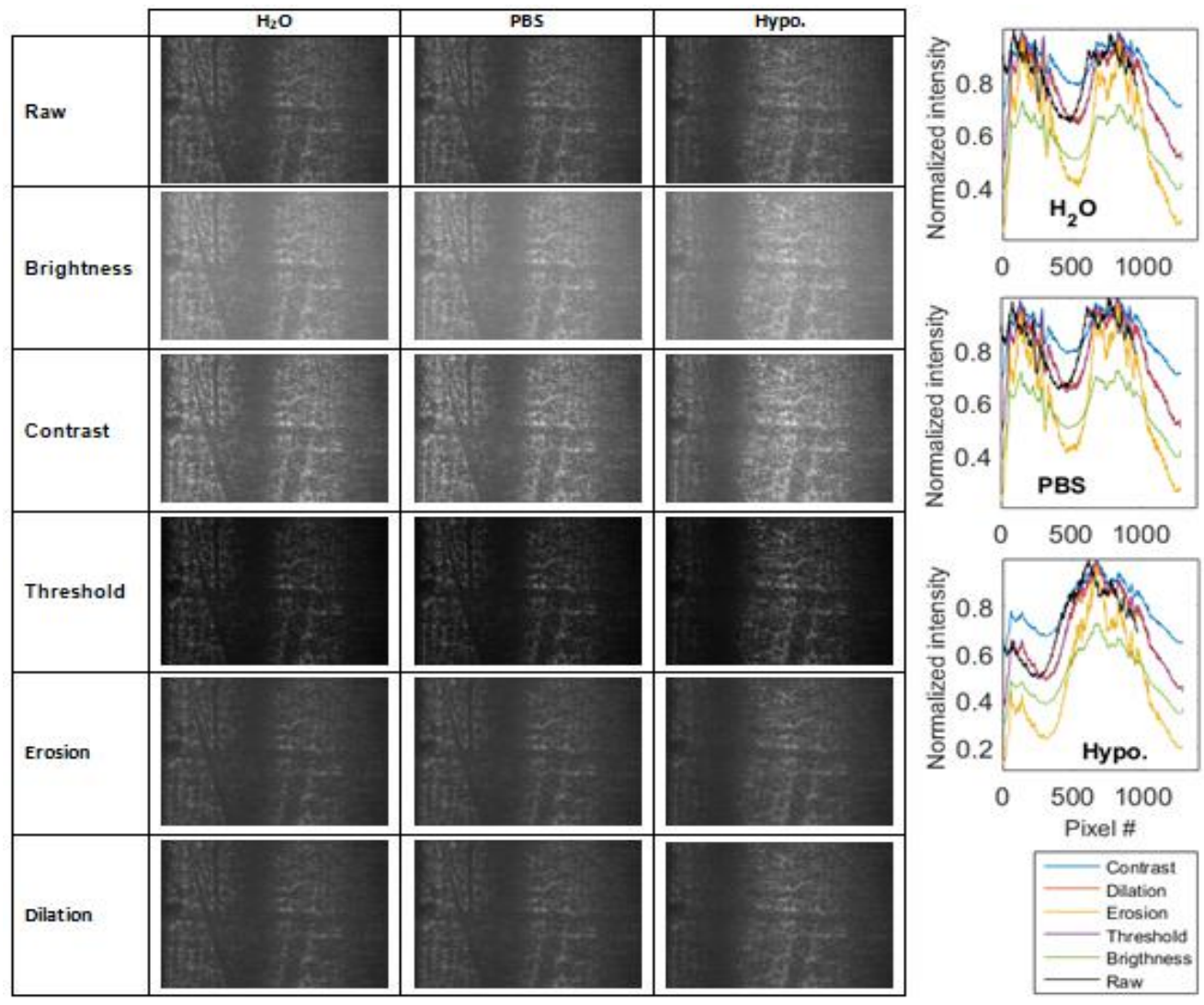

Figure 4 - SPR images of each substances. DPI technique and the obtained SPR curves. Erosion processing gives the highest SPR curve dip, and dos not disturbing the curve features as width, asymmetry and slope [5].

\section{STATISTICAL SPR IMAGE/DATA SEGMENTATION}

The enhanced SPR image with the erosion method is then segmented into several regions $(\mathrm{Si})$ to obtain the best SPR curve. An image segmentation process can be used to remove degradations/imperfections, and to perform a multispot sensing strategy $[3,15]$. The statistical segmentation allows a reorganization of pixels to obtain a better dark region/dip of the resonance position. The bootstrap statistical method [17] was used in SDS. The basic idea of this method is to estimate the desired characteristics for the SPR curve, allowing to approximate the desired pixel distribution of the image by an empirical distribution based on a finite-size sample (B). The bootstrap enables greater flexibility, speed, and fewer assumptions in determining estimates. The sampling is performed with replacement [21]. Confidence intervals within the region of interest are generated, allowing inferences (significance tests), and information extraction about minimum points in the images of each analyzed substance.

A sample is statistically significant for $B \geq 2000$ [17,21]. As illustrated in Fig. 5a, the $\alpha$ confidence interval is defined in which the upper (SUP) and lower (INF) limits delimit the percentiles of the bootstrap estimate that will be used to represent the new SPR curve. Here, the SDS was investigated for 0.01, 0.05 and 0.1 intervals in samples of sizes 2000, 4000 and 8000.

Fig. $5 b$ shows the resonance position $\left(\theta_{R}\right)$ extracted from the new SPR curves obtained with the SDS. For the pair $\alpha=0.05$ and $B=2000$ it is possible to obtain the greatest variation in the resonant position among the analyzed substances (see Fig. 5c). 


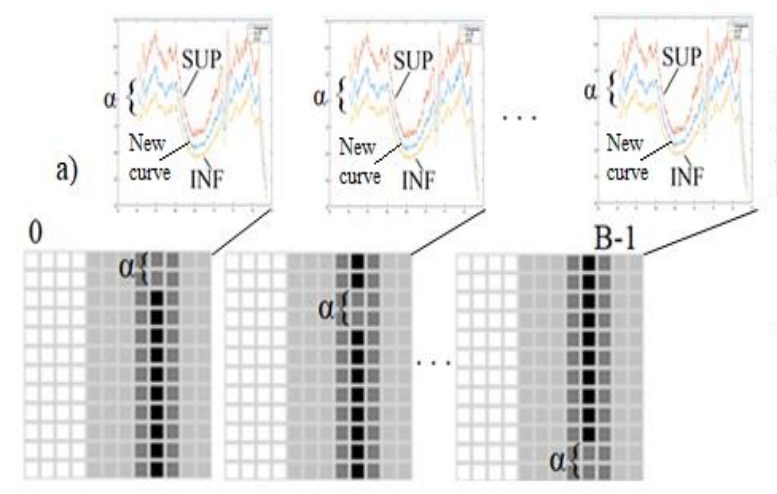

\begin{tabular}{|c|c|c|c|}
\hline & \multicolumn{3}{|c|}{$B=8000$} \\
\hline$\alpha$ & $\mathrm{H}_{2} \mathrm{O}$ & PBS & Hypo. \\
\hline 0.01 & $69.07^{\circ}$ & $69.45^{\circ}$ & $70.62^{\circ}$ \\
\hline 0.05 & $68.98^{\circ}$ & $69.45^{\circ}$ & $70.62^{\circ}$ \\
\hline 0.1 & $68.98^{\circ}$ & $69.45^{\circ}$ & $70.62^{\circ}$ \\
\hline \multirow{8}{*}{ b) } & \multicolumn{3}{|c|}{$B=4000$} \\
\hline & $69.11^{\circ}$ & $69.45^{\circ}$ & $70.62^{\circ}$ \\
\hline & $69.11^{\circ}$ & $69.45^{\circ}$ & $70.62^{\circ}$ \\
\hline & $69.11^{\circ}$ & $69.45^{\circ}$ & $70.62^{\circ}$ \\
\hline & \multicolumn{3}{|c|}{$B=2000$} \\
\hline & $68.94^{\circ}$ & $69.32^{\circ}$ & $70.62^{\circ}$ \\
\hline & $68.94^{\circ}$ & $69.92^{\circ}$ & $70.65^{\circ}$ \\
\hline & $68.94^{\circ}$ & $69.32^{\circ}$ & $70.61^{\circ}$ \\
\hline
\end{tabular}

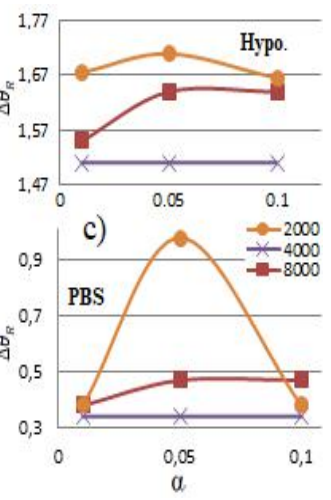

Figure 5 - a) Illustration of the resampling by bootstrap method computed for B samples and statistics $\alpha$ define the confidence intervals. b) Resonance position $\theta_{R}$ obtained from new SPR curves obtained with different values of $B$ and $\alpha$, and c) graphs for resonance variation $\Delta \theta_{R}$ between $\mathrm{H}_{2} \mathrm{O} \rightarrow$ Hypo and $\mathrm{H}_{2} \mathrm{O} \rightarrow$ PBS plotted from the Fig.5 (b) data.

Table 1 summarize resonance angles and sensitivity values for the DIP and SDS combined application. Manual segmentation in two (512 lines) and four (256 lines, see Fig. 1d) were performed to compare with the bootstrap. The range of refractive index (RI) between $\mathrm{H} 2 \mathrm{O} \rightarrow$ Hypo. is $1.35 \mathrm{RIU}$, and $1.5 \mathrm{RIU}$ between $\mathrm{H} 2 \mathrm{O} \rightarrow \mathrm{PBS}$. This corresponds to an angle variation of $0.98^{\circ}$ and $1.71^{\circ}$, respectively, for the DIP (erosion) + SDS (bootstrap: $\alpha=0.05$ and $\mathrm{B}=2000$ ) processed images. The values of angular sensitivity $S_{\theta}$, expressed as the ratio of resonance angle variation $\left(\Delta \theta_{R}\right)$ by the refractive index changes $(\Delta R I)$, i.e., $S_{\theta}=\Delta \theta_{R} / \Delta R I$, reaches $390^{\circ} / \mathrm{RIU}$ for the proposed approach. The performance obtained with the DPI+SDS approach increase $28 \%$ related to manual segmentation of $1 / 4$ of the SPR image, and $81 \%$ to the raw image.

Table 1 - Summarized of the sensing parameter $\theta_{R}$ and correspondent sensitivities. SDS method is applied at DIP erosion processed image.

\begin{tabular}{|c|c|c|c|c|c|c|}
\hline \multirow{3}{*}{$\mathrm{DIP}$} & & & $\mathbf{H}_{2} \mathbf{O}$ & PBS & Нypo. & $\mathrm{S}_{\theta}(\% / \mathbf{R I U})$ \\
\hline & \multicolumn{2}{|l|}{ Raw image } & $68.01^{\circ}$ & $68.58^{\circ}$ & $68.67^{\circ}$ & 214.44 \\
\hline & \multicolumn{2}{|l|}{ Erosion } & $68.96^{\circ}$ & $69.43^{\circ}$ & $70.59^{\circ}$ & 216.74 \\
\hline \multirow{11}{*}{ SDS } & \multicolumn{6}{|l|}{ Manual segmentation } \\
\hline & \multirow{2}{*}{ Two parts } & S1 & $69.60^{\circ}$ & $69.49^{\circ}$ & $71.01^{\circ}$ & 89.44 \\
\hline & & S2 & $69.65^{\circ}$ & $69.93^{\circ}$ & $71.07^{\circ}$ & 143.37 \\
\hline & \multirow{4}{*}{ Four parts } & S1 & $69.51^{\circ}$ & $69.49^{\circ}$ & $71.04^{\circ}$ & 61.44 \\
\hline & & S2 & $69.60^{\circ}$ & $69.72^{\circ}$ & $71.01^{\circ}$ & 91.77 \\
\hline & & S3 & $69.22^{\circ}$ & $69.93^{\circ}$ & $71.07^{\circ}$ & 304.37 \\
\hline & & S4 & $69.73^{\circ}$ & $69.87^{\circ}$ & $71.05^{\circ}$ & 95.66 \\
\hline & \multicolumn{6}{|c|}{ Bootstrap sampling (automatic segmentation) } \\
\hline & \multicolumn{2}{|l|}{$\alpha=0.05 ; \mathrm{B}=2000$} & $68.94^{\circ}$ & $69.92^{\circ}$ & $70.65^{\circ}$ & 390.0 \\
\hline & \multicolumn{2}{|l|}{$\alpha=0.05 ; \mathrm{B}=4000$} & $69.11^{\circ}$ & $69.45^{\circ}$ & $70.62^{\circ}$ & 169.25 \\
\hline & \multicolumn{2}{|l|}{$\alpha=0.05 ; \mathrm{B}=8000$} & $68.98^{\circ}$ & $69.45^{\circ}$ & $70.62^{\circ}$ & 217.40 \\
\hline
\end{tabular}

\section{CONCLUSIONS}

An approach for processing the response of SPR and SPRi sensors based on spatial domain processing of the SPR image, combined with the statistical segmentation of image pixel values was presented. The dip accentuation of the SPRc and visual aspects of SPRi was 
obtained with the application of a morphological erosion filter. Erosion thins objects in an image, accentuating the region relative to the resonance position. However, with this fine-tuning, a displacement of the resonance position is noted after the operation. A statistical segmentation was performed using the bootstrap method. The bootstrap acts in a reorganization of the SPR image pixels to obtain a better SPR curve/image, avoid manual segmentation. The resampling with parameters $\alpha=0.05 ; \mathrm{B}=2000$ showed a significant improvement in the sensitivity value, attesting to the good performance of the proposed DPI + SDS approach.

Author Contribution All authors contributed to the study conception and design. Gleice M. de Medeiros: Software development, Investigation, Data visualization, Validation. Leandro C. de Souza: Development, Reviewing and editing, SDS specialist. Daniel F.L. de Souza: Development, Reviewing and editing, DIP specialist. Leiva C. Oliveira: Supervision, Conceptualization, Development, Writing - original draft, Writing- review \& editing, SPR specialist. All authors commented on previous versions of the manuscript and read and approved the final manuscript.

Funding This work was supported by the Universidade Federal Rural do Semi-Árido (UFERSA) (Grant No. PIC10025-2019 / 23091.014601/2019-77).

Data Availability The data and material used in this work is available from the corresponding author upon reasonable request.

Code Availability The code used during the current study is available from the corresponding author on reasonable request.

\section{DECLARATIONS}

Research Involving Human and Animal Participants This is the original work. No human/animal subject involved/participated in this study.

Consent for Publication No human subject involved in this study. It is the original manuscript that has not been published before, and will not be submitted elsewhere before a decision is made by this journal. So, kindly do the needful for the same.

Conflict of Interest The authors declare that they have no competing interests.

\section{REFERENCES}

[1] J. Homola; et al., Surface plasmon resonance sensors: review. Sensors Actuators B: Chem, v. 54, p. 3$15,1999$.

[2] R.B.M. Schasfoort; A.J. Tudos, Handbook of Surface Plasmon Resonance. The Royal Society of Chemistry, RSC Publishing, Cambridge, 2008.

[3] L.C. Oliveira; et. al. Surface Plasmon Resonance Sensors: A Materials Guide to Design, Characterization, Optimization, and Usage, 2nd ed. Springer, Berlin, 2019.

[4] Mihaela P.; Camelia B., SPR and SPR Imaging: Recent Trends in Developing Nanodevices for Detection and Real-Time Monitoring of Biomolecular Events, Sensors, v. 16, p. 870, 2016.

[5] S. Scarano; et al., Surface plasmon resonance imaging for affinity-based biosensors. Biosensors \& Bioelectronics. v. 25(5), p.957, 2010.

[6] T. de Sousa, et. al., Evaluating the signal processing chain employed in surface plasmon resonance biosensing, In: I2MTC Proceedings, v. 1, p. 1606-1611, 2014. 
[7] E.G. Pereira; et al., Improved data extraction algorithm for biosensors utilizing surface plasmon resonance sensing. In: I2MTC Proceedings, v. 1, p. 884, 2014.

[8] Dongping W.; et al., Recent Advances in Surface Plasmon Resonance Imaging Sensors, Sensors, v. 19(6), p. 1266, 2019.

[9] S. Scarano; et al., Surface plasmon resonance imaging (SPRi)-based sensing: A new approach in signal sampling and management, Bios. \& Bioelect., v. 26(4), p.1380-5, 2010.

[10] Boecker, D.; et al., Noise reduction by multiple referencing in surface plasmon resonance imaging. Rev. Sci. Instrum., v. 79, n. 023110-1, 2008.

[11] J.C.S. Baptista; et al., Smart noise reduction in SPR sensors response using multiple-ANN design. IEEE Sensors Journal, v. 21, p. 4517 - 4524, 2020.

[12] I.N. Pavlov; et al., The choice of an image processing algorithm for increasing sensitivity of the surface plasmon resonance method, J. Phys.: Conf. Ser., v. 1421, 2019.

[13] Srestha S., Image denoising using new adaptive based median filter Signal and Image, Processing: an International Journal, v. 5(4), p. 13, 2014.

[14] Gonzalez, R.; Woods, R. Digital Image Processing, 3rd ed, Pearson, Prentice Hall, 2008.

[15] L.C. Oliveira; et al., SPR sensor for tampering detection in biofuels. In: I2MTC Proceedings, v. 1, p. 1471-1476, 2015.

[16] S. Bayani; et al., High-Speed Surface Plasmon Resonance Imaging and Image Processing Method, J. Flow Vis. Image Proc., v. 25(3-4), p. 191-205, 2018.

[17] Efron, R. T. B. An Introduction to the Bootstrap, Chapman and Hall, 1993.

[18] L.C. Oliveira; et al., A Prism-based Polymeric Surface Plasmon Resonance Biochip for Angular and Spectral Modes, Procedia Engineering, v. 168, p. 1350-1353, 2016.

[19] L.C. Oliveira; et al., A surface plasmon resonance biochip that operates both in the angular and wavelength interrogation modes, IEEE TIM, v. 62, n. 5, p. 1223-1232, 2013.

[20] G. Guekos and M.J. O Strutt, IEEE J. Quantum Electron., v. 6, p. 423, 1970.

[21] MathWorks, Help Center website: https://www.mathworks.com/help/stats/bootstrp.html Access in Oct 2021. 